\title{
La conception de l'offre formation professionnelle au Maroc Approche marketing
}

\author{
Habib Benaissa
}

\begin{abstract}
Résumé: Les attentes des usagers et l'évolution des conditions économiques ont révolutionné la gestion du savoir. La conception des acquisitions du savoir doit donc donner lieu à l'élaboration de nouvelles stratégies basées sur une compréhension approfondie des problèmes pour proposer une nouvelle orientation pour le système éducatif et l'adapter aux besoins de la nouvelle économie et du marché de l'emploi. L'analyse des besoins permet de faire ressortir les enjeux mais aussi les difficultés qui causent l'insatisfaction à la fois des usagers et du milieu de travail. Elle sert aussi, à repérer les manques. Le résultat de cette analyse permettra de mieux cerner toutes les questions pertinentes à ce sujet, tel que le développement et la modification de prestations de formation, en se focalisant sur les questions et les décisions les plus importantes pour procéder à une conception professionnelle d'un produit de formation crédible, pertinent et utile pour ses consommateurs. Il s'agit d'élaborer une démarche concrète qui répond aux différentes dimensions d'une situation de manière à s'assurer qu'elle remplissait bien le mandat crucial qui lui était conféré à cet égard. Au Maroc, les opérateurs économiques sont unanimes sur le besoin d'une formation mutante qui s'adapte davantage avec le marché du travail.
\end{abstract}

Mots clés: Formation professionnelle, analyse des besoins, marchés du travail.

\section{Introduction}

Dans un environnement durablement compétitif et fortement concurrentiel, former les personnes est devenue un levier important pour la productivité, la compétitivité, la croissance économique des pays, l'insertion des jeunes dans la vie active et la lutte contre le chômage.

En effet, développer les compétences est devenu un aspect incontournable pour au moins deux considérations ; D'abord, parce que les compétences deviennent un facteur déterminant dans un environnement économique instable qui nécessite des innovations continues. Ensuite, permettre aux individus de développer leur portefeuille de compétences en vue d'une meilleure professionnalisation. Cette problématique a mis la pression sur les entreprises et les établissements de formation, qui sont contraints de travailler dans un environnement marqué par une forte fluctuation des marchés et une concurrence accrue, ce qui a placé la formation aujourd'hui au cœur des enjeux économiques et sociaux de notre société.

Les expériences et les concepts de la planification de la formation professionnelle au Maroc ont connu des changements substantiels depuis l'année 2000. Plusieurs probabilités qui visaient la rationalisation du processus du développement de la formation professionnelle se sont révélées inadéquates et ont été critiquées ou abandonnées. La nécessité d'études approfondies et d'une responsabilisation accrue en vue d'établir des critères permettant de générer des données comparables, de mesurer l'efficacité des produits formation en vigueur, de mener des études plus sectorielles et thématiques, d'appliquer des critères homogènes de concentration apparait comme une solution pertinente pour concevoir des produits qui répondront aux exigences des consommateurs de la formation. Ceci suscite une double question qu'on peut formuler ainsi : Le savoir est-il un client et la formation est-elle un bien de consommation comme les autres ?

Dans le premier axe de cet article, nous donnons un aperçu sur la situation actuelle de la formation professionnelle au Maroc qui a connu de multiples transformations tout au long de son évolution. Le deuxième axe sera exclusivement réservé à l'offre de la formation professionnelle au Maroc, ses besoins et ses finalités, puisque, la conception de tout produit formation s'articule sur deux étapes primordiales : examiner les besoins et fixer les finalités, car toute politique nouvelle visant à réformer le système de formation échouera si elle ne tient pas compte des finalités explicites et adéquates avec les besoins exprimés. Nous allons tenter dans le troisième axe de cet article de développer quelques approches théoriques sur la notion de professionnalisation de la formation professionnelle qui se trouve au centre de l'approche procédurale, à un moment où le terme formation est devenu plus courant que l'éducation obéissant à une perception utilitaire qui se décline vers le professionnalisme. La formation fait souvent allusion aux termes, "Professionnalisation », "parcours professionnalisant», "professionnaliser », qui sont devenus, une sorte de gage de légitimité des actions de formation ce qui implique d'articuler le monde de l'éducation à celui de l'économie. 


\section{1- Définition et enjeux de la formation}

\subsection{Définition}

Étymologiquement, former du latin (formare) c'est donner une forme, c'est créer ou réaliser ce qui n'existe pas. La formation est littéralement cette action de faire apparaître quelque chose. Elle deviendra, par la suite, l'action de former quelqu'un intellectuellement ou moralement par le biais de l'instruction et de l'éducation. La formation, comme institution, fait désormais partie intégrante de notre société, notre vie et de notre histoire. (Ardouin T., 2008) ${ }^{1}$. Deux années plutôt, il a considéré la formation comme une "action de se doter de moyens pour permettre l'acquisition de savoir par une personne ou un groupe, dans une perspective contractuelle, en lien avec un contexte donné en vue d'atteindre un objectif. »(Ardouin T. 2006) ${ }^{2}$.

La formation professionnelle s'adresse aux personnes souhaitant maitriser un " savoir faire » pour obtenir un diplôme et s'insérer sur le marché du travail. «Elle vise l'acquisition des compétences qui permettent à la personne d'assumer son rôle de travailleur et d'évoluer sur le marché de l'emploi. La formation professionnelle vise également à répondre aux exigences de la société. En ce sens, elle tient compte de la structure socioéconomique, des besoins de ce monde du travail, des politiques de développement de la maind'œuvre ainsi que des caractéristiques de chaque métier. De plus, la formation professionnelle permet à la personne de contribuer au développement technologique ainsi qu'à la croissance culturelle et socioéconomique de son milieu. »(Organisation Internationale de la Francophonie.2010)․

A travers ces définitions, il apparait clairement que l'éducation et la formation sont des besoins fondamentaux qui touchent à de multiples aspects la vie des individus et des communautés, comme l'a souligné Danton en $(1793)^{4}$ « Après le pain, l'éducation est le premier besoin d'un peuple. »

En effet, un besoin de formation est défini comme un écart entre «ce qui est» et « ce qui devrait être », entre une situation actuelle et une situation désirée, dû à une absence ou à un manque de compétences essentielles ou requises. (Nous reviendrons sur ce point par la suite d'une manière, relativement, plus détaillée).

\subsection{Situation actuelle du dispositif national de la formation professionnelle}

Conscient de l'enjeu que représente la formation des jeunes et leur accompagnement vers l'emploi à travers une production de compétences mieux ciblées, Le Maroc, a mit en œuvre des mesures pour assurer le développement de compétences en renforçant la formation professionnelle initiale et la promotion de la formation continue. Dés lors, la formation professionnelle bénéficie d'un intérêt croissant par le gouvernement marocain qui a mené de nombreuses expériences et des réformes institutionnelles plus vastes visant la refonte de l'ensemble du secteur et du dispositif de formation professionnelle, telles que l'approche par compétence, progressivement étendue depuis 2003, les enquêtes d'insertion des lauréats, ententes entre l'Etat et des entreprises pour la gestion des centres, détermination des secteurs principaux et des places ouvertes par filière, etc. En effet, des efforts importants été consentis en matière de financement. Le budget global de la formation professionnelle au Maroc s'est élevé en 2010, à 0,48 \% du PIB (Organisation Internationale de la Francophonie, 2010) financé en grande partie par l'Etat $(74 \%$, y compris par la TFP, versée par les entreprises) et les ménages $(21 \%)$. Les dépenses de fonctionnement représentent une partie prépondérante de ce budget (77\%, essentiellement les salaires). Le dispositif de formation professionnelle est composé de cinq degrés, comme indiqué dans le tableau 1.

Tableau 1. Les cinq niveaux de la formation professionnelle au Maroc et évolution de l'effectif des stagiaires par niveau de formation

\begin{tabular}{|c|c|c|}
\hline Niveau de Formation & $2013 / 2014$ & $2014 / 2015$ \\
\hline Technicien Spécialisé & 105305 & 119634 \\
\hline Technicien & 109362 & 123264 \\
\hline Qualification & 75277 & 81126 \\
\hline Spécialisation & 53209 & 60316 \\
\hline Certificat de Formation par Apprentissage & 7412 & 6992 \\
\hline Total & 350565 & 391332 \\
\hline
\end{tabular}

Source : Département de la Formation Professionnelle, Rapport d'activités 2015

\footnotetext{
${ }^{1}$ Cité par Ardouin T., (2008). » Formation tout au long de la vie et professionnalisation à l'université : le cas des métiers de la formation à l'université de Rouen ".

${ }^{2}$ ARDOUIN, T. Ingénierie de formation pour l'entreprise. Paris: Dunod, (2006). $2^{\text {ème éd. p10 }}$

${ }^{3}$ Organisation Internationale de la Francophonie, (2010) «Les guides méthodologiques d'appui à la mise en œuvre de

l'approche par compétence en formation professionnelle »

${ }^{4}$ Georges Jacques Danton, (1973) «Discours à l'Assemblée législative, 13 août 1793. »

${ }^{5}$ Organisation Internationale de la Francophonie, (2010) «LES GUIDES MÉTHODOLOGIQUES D’APPUI À LA MISE EN

CEUVRE DE L'APPROCHE PAR COMPÉTENCES EN FORMATION PROFESSIONNELLE »
} 
Si des bonds en avant suffisants ont été enregistrés depuis les années 2000, il reste bien des efforts à accomplir en faveur de la compétitivité en renforçant le système de formation pour mettre en œuvre un projet pilote permettant de hisser l'enseignement et la formation vers de nouveaux sommets. La situation actuelle est malheureusement, marquée par le manque de coordination entre ministères concernées, entre formation initiale et continue, et entre ces deux dernières et le marché de travail. Le système de formation professionnelle fait l'objet de nombreuses critiques quant à sa trop grande complexité et son inefficacité. De manière générale, la gouvernance du dispositif de formation professionnelle crée un risque de confusion dans l'offre de formation, ou de concurrence entre opérateurs, en même temps qu'elle demande un suivi plus compliqué du coût et de l'efficacité de chaque formation.

En plus du DFP, chargé par un décret de 2005 d'élaborer la politique, d'exécuter et d'évaluer les stratégies, les départements ministériels de l'artisanat, du tourisme, de l'agriculture et des pêches, de l'industrie et du commerce, sont aussi impliqués dans la gouvernance de la formation professionnelle et participent à l'élaboration et au suivi des politiques, l'OFPPT quant à lui joue un rôle décisif dans l'attribution de la taxe professionnelle et le développement de la FPCE.

D'autres organismes participent à la gouvernance de la formation professionnelle, à savoir :

- Le Conseil Supérieur de l'Enseignement (CSE) créé en 2006

- Le Conseil économique, social et environnemental créé en 2011

- Les commissions provinciales de la FP

- Les commissions régionales pour l'amélioration de l'employabilité

- La Fédération marocaine de l'enseignement professionnel privé

- Les commissions sectorielles et régionales de la FP privée

- $\quad$ La CGEM et les syndicats tels que l'UMT, l'UGTM, la CDT, la FDT l'UNT

- Le Comité central des contrats spéciaux de formation

Cette multiplicité des acteurs est une bonne chose mais les activités ne correspondent pas toujours au positionnement institutionnel, ce qui représente une entrave à la bonne élaboration d'une planification stratégique de la formation professionnelle, on a l'impression que les tâches et les attributions ne sont pas reparties de façon homogène et que le DFP n'arrive pas à s'acquitter des tâches qui lui ont été affectées dans l'absence d'une capacité organisationnelle plus fondamentale d'où le flou qui entoure la définition des politiques de la formation professionnelle, l'évaluation des besoins du marché du travail, l'élaboration de la carte prévisionnelle des formations et l'évaluation des établissements de formation professionnelle. Ces insuffisances dues à certaines faiblesses dans la coordination des acteurs et des limitations dans l'efficacité et l'équité du système, à l'absence d'une vision partagée par tous, à l'inadaptation de certains textes juridiques et au manque de mécanismes pertinents de pilotage et d'évaluation, comme l'a démontré le travail préparatoire à l'élaboration de la stratégie nationale de la FP conduit en 2012/13.(Fondation européenne pour la formation, 2014) ${ }^{6}$

\subsection{Ouverture, qualification et accréditation des établissements de formation professionnelle}

Les procédures d'ouverture, de qualification et d'accréditation des établissements de formation professionnelle se caractérisent par l'absence de planification ce qui laisse supposer qu'il existe un manque de cohérence, d'harmonie et de bonne entente entre les acteurs.

On remarque que ces procédures varient en fonction des types d'EFP. Les établissements de l'OFPPT sont créés par arrêté du Ministère de l'éducation et de la formation professionnelle après une assertion du conseil d'administration. Ceux des ministères formateurs dépendent d'un arrêté du ministère concerné et d'un visa du Ministère de l'éducation et de la formation professionnelle. Tous les diplômes de ces établissements sont pratiquement reconnus au plan national dès l'émission de l'arrêté d'ouverture.

La création d'un établissement de formation professionnelle privée est soumise aux dispositions de la loi 13.00 portant statut de la formation professionnelle privée et à son décret d'application $\mathrm{n}^{\circ} 2.00 .1018 \mathrm{du} 21$ juin 2001. Le promoteur du projet est tenu à préparer un dossier de demande et le déposer au DFP suite à un avis de la commission régionale interprofessionnelle de la formation professionnelle privée, après l'obtention d'un accord de principe, il doit ensuite l'entériner sous forme d'un procès verbal d'adhésion par la délégation régionale du DFP. Pour finaliser sa demande, l'intéressé est tenu à préparer une étude de faisabilité, un projet pédagogique détaillé, un dossier administratif et un projet de règlement intérieur. Pour que les diplômes fournis par un EPFP soient reconnus, le dirigeant doit d'abord, assurer la formation d'au moins une promotion de lauréats dans la filière proposée à la qualification, ensuite l'accréditation de l'EPFP est délivrée pour au moins

\footnotetext{
${ }^{6}$ Fondation européenne pour la formation, 2014 (GEMM GOUVERNANCE POUR L'EMPLOYABILITÉ EN MÉDITERRANÉE) «CARTOGRAPHIE DE LA GOUVERNANCE DE LA FORMATION PROFESSIONNELLE AU MAROC » 
une période de trois ans mais qui nécessite la qualification de l'ensemble des filières. Ces démarches compliquent et ralentissent les procédures d'ouverture, de qualification et d'accréditation.

En effet, cette gouvernance déficiente pose problème aussi bien pour les EPFP qu'aux usagers. Malgré une prise de conscience croissante par tous les acteurs des enjeux et du besoin de faire évoluer la formation professionnelle vers les priorités de développement économique et sociale, les rôles et attributions des différents acteurs ne sont malheureusement ni déterminés ni clarifiés. Cette procédure est marquée par la diversité des intervenants impliqués dans la mise en œuvre de la formation professionnelle, les défectuosités des textes et le retard de leurs adaptations aux pratiques émergentes et évolutives empêchent l'efficacité du pilotage.

Le projet GEMM (2014) ${ }^{7}$ de la Fondation Européenne pour la formation a dressé un diagnostic rigoureux et approfondi des forces et faiblesses du système de la formation professionnelle au Maroc dont ci-dessous les détails :

Tableau 2 Analyse Du Comité De Pilotage Du Projet

\begin{tabular}{|c|c|}
\hline Points forts & Points faibles \\
\hline $\begin{array}{l}\text { - Tous les acteurs sont conscients du rôle et des enjeux de la } \\
\text { FP pour le développement économique et social du pays. } \\
\text { - Cette conscience est présente dans le discours politique du } \\
\text { gouvernement, des partenaires sociaux et économiques. }\end{array}$ & $\begin{array}{l}\text { - Les rôles et responsabilités des différents intervenants ne sont } \\
\text { ni définis ni clarifiés. } \\
\text { absence de visibilité sur le financement de la FP et les règles } \\
\text { et procédures d'allocation de ressources et d'évaluation des } \\
\text { résultats. } \\
\text { absence d'un système d'information intégré opérationnel } \\
\text { nécessaire à la définition de la politique de formation. }\end{array}$ \\
\hline Opportunités & Menaces \\
\hline $\begin{array}{l}\text { Améliorer la transparence et l'équité dans l'allocation et } \\
\text { l'utilisation des financements. } \\
\text { a Renforcer le rapprochement de l'offre de formation de la } \\
\text { demande économique et sociale. } \\
\text { a Redynamiser et élargir la concertation et la participation } \\
\text { des parties prenantes (au niveau national et territorial) dans } \\
\text { la définition, la mise en ouvre et l'évaluation des politiques } \\
\text { de formation. } \\
\text { - Nouvelle architecture du gouvernement (Ministère de } \\
\text { l'Education Nationale et de la Formation Professionnelle) } \\
\text { favorisant la coordination et la complémentarité }\end{array}$ & $\begin{array}{l}\text { - L'émergence de plusieurs politiques non coordonnées et non } \\
\text { complémentaires. } \\
\text { a Dispersion des efforts et des moyens. } \\
\text { a Consécration de la situation de non-reddition des comptes et } \\
\text { de non transparence. } \\
\text { a Surcoûts entraînés par la non qualité et l'inadéquation des } \\
\text { formations. }\end{array}$ \\
\hline
\end{tabular}

Source : projet de gouvernance pour l'employabilité dans la région méditerranéenne (GEMM) 2014.

La politique marocaine de la formation professionnelle cible à la fois le développement de la personne et la réponse aux impératifs de développement économique. Ce double objectif incite l'État à définir les finalités à avantager, en tenant compte de l'articulation retenue, notamment, lors de l'élaboration des produits formation. L'organisation de formation s'articule sur deux étapes primordiales : examiner les besoins et fixer les finalités, car toute politique nouvelle visant à réformer le système de formation échouera si elle ne tient pas compte des finalités explicites et adéquates avec les besoins exprimés.

\section{Connaitre les besoins et les finalités de la formation professionnelle}

Les besoins de formation peuvent être individuels ou collectifs, (Fernandez 1988, $)^{8}$ estime que « le besoin se définit comme ce qui est indispensable ou, du moins, utile à l'organisation ou aux individus pour atteindre un objectif valorisé et justifiable».

Toutes les définitions fiables décrivent le besoin comme un «écart » entre une situation souhaitée et optimale, et une situation existante et déséquilibrée, Déjà en 1950, Tyler considère que "Les études portant sur les apprenants suggèrent des objectifs opérationnels seulement lorsque les informations recueillies sont comparées à des standards désirés, à des normes jugées acceptables, de telle sorte que la différence entre la condition actuelle de l'apprenant et la norme acceptable peut être identifiée ». (Tyler 1950) ${ }^{9} »$. Kaufman $(1982)^{10}$ lui, emboite le pas à Tyler et affirme que le besoin est un écart entre «ce qui est et ce qui devrait être », et ce, en terme de résultats.

La formulation des besoins renvoie dans un même temps aux demandes de besoins (j'ai des besoins) ou aux offres des besoins (je crée des besoins).

\footnotetext{
${ }^{7}$ Fondation européenne pour la formation, 2014 (GEMM GOUVERNANCE POUR L'EMPLOYABILITÉ EN MÉDITERRANÉE) « CARTOGRAPHIE DE LA GOUVERNANCE DE LA FORMATION PROFESSIONNELLE AU MAROC »

${ }^{8}$ FERNANDEZ, J. (1988). « Réussir une activité de formation », p 57.

${ }^{9}$ Tyler, R.W. (1950). Basic Principles of Curriculum and Instruction, p 5-6

${ }^{10}$ Kaufman, R.E. (1982). Identifying and Solving Problems, p 73

DOI: $10.9790 / 487 X-1904024658 \quad$ www.iosrjournals.org $\quad 49 \mid$ Page
}


Tableau 3. Analyse Des Demandes Et D'offres Des Besoins

\begin{tabular}{|c|c|c|c|}
\hline \multicolumn{2}{|c|}{ Les besoins en tant que demandes } & \multicolumn{2}{|c|}{ Les besoins en tant qu'offres } \\
\hline \multicolumn{2}{|c|}{$\begin{array}{l}\text { Entre la société à l'individu il existe un large éventail de } \\
\text { niveaux de demandes en matière d'éducation et de formation }\end{array}$} & \multicolumn{2}{|c|}{$\begin{array}{l}\text { Emettre une offre de formation peut répondre à diverses } \\
\text { finalités. }\end{array}$} \\
\hline Demandeurs & Besoins & Emetteurs & Besoins \\
\hline L'Etat & $\begin{array}{l}\text { Chaque année, l'Etat manifeste des } \\
\text { besoins en matière de personnel, en } \\
\text { termes de recrutement pour combler les } \\
\text { besoin en ressources humaines }\end{array}$ & L'Etat & $\begin{array}{l}\text { Anticipation d'un besoin en compétences } \\
\text { - préparer les spécialistes et les former aux } \\
\text { métiers de demain } \\
\text { - adapter la main d'œuvre aux mutations } \\
\text { industrielles } \\
\text { - anticiper les tendances du marché du } \\
\text { travail et des besoins en qualification }\end{array}$ \\
\hline $\begin{array}{l}\text { Entreprise, } \\
\text { organisation }\end{array}$ & $\begin{array}{l}\text { Besoins en formation initiale en terme } \\
\text { de : } \\
\text { Compétences à jour et des personnels } \\
\text { dotés de nouvelles compétences } \\
\text { spécialisées nécessaires pour faire face } \\
\text { aux besoins changeants en terme } \\
\text { d'emploi } \\
\text { Besoins en formation continue pour : } \\
\text { Renforcer et/ou améliorer les } \\
\text { compétences de leurs employés }\end{array}$ & EFP & $\begin{array}{l}\text { Les offres des EFP destinées au marché de } \\
\text { l'emploi, aux besoins en compétences des } \\
\text { entreprises et aux projets professionnel de } \\
\text { la personne prennent en considération les : } \\
\text { - besoins liés à la stratégie de l'entreprise } \\
\text { - besoins liés aux profils des usagers } \\
\text { apprenants } \\
\text { - besoins liés aux milieux du travail }\end{array}$ \\
\hline Un individu & $\begin{array}{l}\text { - Actualiser ses connaissances ; } \\
\text { - Développer de nouvelles compétences } \\
\text { et une plus grande autonomie ; } \\
\text { - S'adapter aux évolutions } \\
\text { technologiques, organisationnelles ou } \\
\text { socio-économiques ; } \\
\text { - Acquérir une nouvelle qualification; } \\
\text { - Se préparer à un changement } \\
\text { d'activité ; Se maintenir dans } \\
\text { l'emploi. }\end{array}$ & Entreprise & $\begin{array}{l}\text { Pour faire face aux besoins de formation } \\
\text { prioritaires l'entreprise offre des formations } \\
\text { permanentes à ses salariés }\end{array}$ \\
\hline
\end{tabular}

Source : moi-même

Généralement, les offres de formation reflètent les finalités de la formation professionnelle, à savoir :

- Permettre à la personne de conserver son indépendance et son objectivité dans l'exercice de ses fonctions dans un domaine d'activité professionnelles et concourir à son épanouissement.

- Faciliter une meilleure appropriation des compétences en qualité et en quantité, permettant de développer l'autonomie et l'esprit du groupe et d'entreprise pour répondre aux exigences du marché de l'emploi souvent renouvelé ; aider les individus à se sentir utiles dans la société et participer au progrès économique, social et culturel ; soutenir les apprenants à acquérir une forte personnalité et accroître leur sentiment de sécurité et leur autonomie.

La rédaction des finalités d'une formation doit donc, être fondée sur l'évaluation, la définition des besoins et des priorités qui, de leurs côtés, doivent être traités pour déterminer les finalités auxquelles chaque phase d'une formation devra se référer, dans le but de rendre l'usager un professionnel autonome, responsable et réflexif, c'est-à-dire capable d'analyser toute situation relative à son métier, d'avoir les facultés et la capacité de décider par lui-même pour développer une pensée créative et opérationnelle.

Les finalités de la formation professionnelle fixent le but recherché dans une formation en termes de compétence, concept qui sous-tend, les connaissances, les habilités et l'expérience susceptibles d'être utilisées dans un contexte donné ayant les attitudes appropriées.

Cette notion comporte deux volets, l'un renvoi à la définition des finalités de la formation professionnelle en tant que produit et l'autre exige la détermination des finalités de la formation professionnelle en tant que processus que nous pouvons illustrer ainsi :

Tableau 4. Les finalités de la formation professionnelle

\begin{tabular}{|l|l|}
\hline En tant que produit & En tant que processus \\
\hline $\begin{array}{l}\text { Acquérir le savoir et les savoir-faire (capacité et aptitude) } \\
\text { nécessaires à l'exercice d'un métier ou d'une activité } \\
\text { professionnelle. }\end{array}$ & $\begin{array}{l}\text { Assurer l'acquisition qualitative et quantitative des } \\
\text { compétences nécessaires pour répondre aux besoins actuels et } \\
\text { futurs du marché du travail }\end{array}$ \\
\hline Décrocher un emploi raisonnable et en faire une carrière & $\begin{array}{l}\text { Répondre aux besoins du marché du travail et de l'économie en } \\
\text { ressources humaines qualifiées, afin de contribuer à } \\
\text { l'amélioration de la compétitivité et de la performance des } \\
\text { entreprises }\end{array}$ \\
\hline Obtenir un avancement professionnel & $\begin{array}{l}\text { contribuer à l'innovation, à la créativité, à la modernisation, et à } \\
\text { la compétitivité de tous les secteurs de l'économie }\end{array}$ \\
\hline
\end{tabular}




\begin{tabular}{|l|l|}
\hline $\begin{array}{l}\text { Assumer ses responsabilités comme travailleur ou } \\
\text { travailleuse dans un champ donné d'activités professionnelles } \\
\text { et contribuer à son développement }\end{array}$ & $\begin{array}{l}\text { contribuer à la promotion sociale et professionnelle de la } \\
\text { population active }\end{array}$ \\
\hline Développement de sa personne & contribuer au développement social, économique et culturel \\
\hline Augmenter la pertinence de votre CV & \\
\hline
\end{tabular}

Source : moi-même

En outre, l'intégration d'un établissement de formation incite l'usager apprenant à fixer des objectifs pertinents et réalistes, rechercher des possibilités d'apprentissage et choisir parmi les offres disponibles, entrer en contact avec des institutions, des professeurs ou des formateurs, mener à bien le processus d'apprentissage, surmonter des difficultés, rester motivé tout au long de l'apprentissage, etc. Si l'usager ne détient pas de compétences de base et n'être pas pourvu des facultés adéquates, l'agencement et l'application de tous les niveaux de l'apprentissage deviennent vite très difficiles, voire impossibles.

Nul ne peut nier l'importance des compétences clés comme: la lecture, l'écriture, le calcul, l'utilisation d'un ordinateur, mais les compétences de base communes relatives aux compétences personnelles et sociales ainsi que la gestion de soi constituent le noyau dur de tout processus de formation ou de développement personnel. Les responsables de la conception des produits formation sont tenus à rendre à l'école, au collège et au lycée leurs places d'antan qui sont les principales institutions responsable d'assurer la base officielle du savoir.

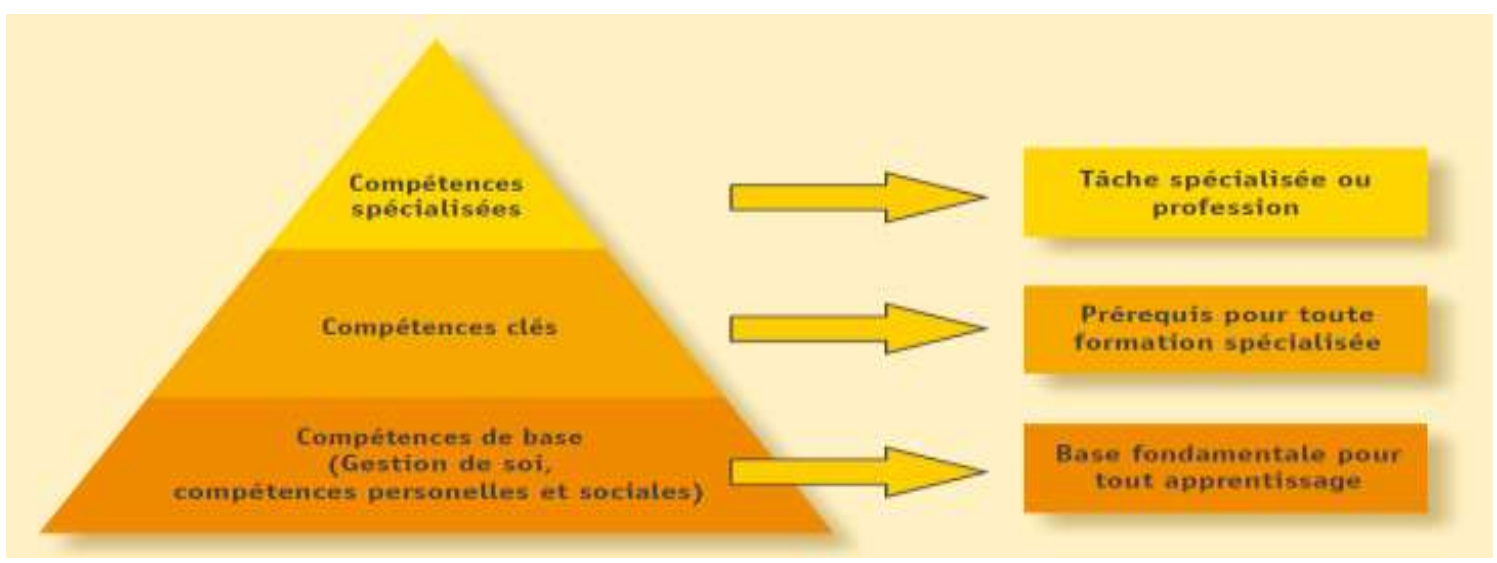

Source : Compétences de base, clés et spécialisées (@ Pro-Skills 2008)

Les savoirs de base appelés communément dans le milieu professionnel « les compétences de base » sont considérées comme des facteurs déterminants qui permettent d'accroitre les « compétences clés », elles regroupent : Compétences linguistiques en français, en particulier dans le cas d'employés qui ont une interaction avec la clientèle: communication orale, communication écrite (lecture, écriture)

- Compétences mathématiques : capacité à manier les nombres, compté, opérer sur des grandeurs

- Compétences cognitives : raisonnement logique, résolution de problèmes, repérage dans l'espace et dans le temps, concentration, mémorisation, etc.

\subsection{Analyse des besoins pour une bonne orientation de formation professionnelle}

L'analyse des besoins permet de faire ressortir les enjeux mais aussi les difficultés qui causent l'insatisfaction à la fois des usagers et du milieu de travail. Elle sert aussi, à repérer les manques. Le résultat de cette analyse permettra de mieux cerner toutes les questions pertinentes à ce sujet, tel que le développement et la modification de prestations de formation, en se focalisant sur les questions et les décisions les plus importantes.

«Toute procédure systématique utilisée pour déterminer des priorités et prendre des décisions eu égard à un programme et à l'allocation de ressources. » est une 'analyse de besoins' selon Witkin (1984) ${ }^{11}$ et d'ajouter « et que cette procédure, qui se veut à la fois objective et reliée à des valeurs, nécessite la collecte et l'analyse de données provenant de diverses sources et demande la considération de différents points de vue. »

L'objectif énoncé d'une analyse de besoins comporte le traitement de deux situations, la situation actuelle de l'étudiant et la situation désirée de l'étudiant, et la détermination de l'écart existant entre les deux. Elle ne peut donc être optimum que si les situations actuelles et futures des usagers peuvent être connues.

\begin{tabular}{|c|c|c|}
\hline DOI: $10.9790 / 487 X-1904024658$ & www.iosrjournals.org & $51 \mid$ Page \\
\hline
\end{tabular}


Nadeau, M.A, $(1988)^{12}$, estime que le processus d'analyse de besoin est essentiellement empirique, ce qui suppose l'acceptation d'un certain nombre d'hypothèses. Elle ne peut être réalisée que si les hypothèses suivantes tiennent :

- La réalité peut être connue, comprise et représentée sous une forme symbolique.

Comme l'analyse de besoins consiste à rendre explicite une réalité, il doit être possible de représenter celleci sous forme symbolique, verbale ou mathématique.

- La réalité n'est pas statique. Par conséquent, l'analyse de besoins doit être un processus continu.

L'évolution des champs de connaissance et de leur application suppose la réévaluation constante des fins de l'éducation.

- Tout peut être mesuré sur une quelconque échelle.

Bien que certains phénomènes éducationnels ne puissent être mesurés sur une échelle à intervalles ou sur une échelle de rapport, ces phénomènes peuvent, à la limite, être représentés à partir d'indices plus grossiers résultant de l'application des échelles nominales ou ordinales ;

- Les fins ou les buts de l'éducation peuvent être précisés.

Il existe un ensemble de moyens permettant aux éducateurs, d'une part, d'expliciter les buts de l'éducation, et, d'autre part, d'obtenir des consensus au niveau de ces buts.

Les éléments nécessaires qui ont une influence sur la bonne exécution de tout produit de formation efficace sont notamment l'adéquation entre celui-ci et les besoins du marché de l'emploi et une collaboration avec le secteur privé, les syndicats, les associations, les autorités locales et le pouvoir central. D'où la nécessité de développer une stratégie d'orientation de la formation vers le développement de compétences et aux demandeurs d'emploi peu qualifiés, mais aussi vers la mise en place d'une main-d'œuvre possédant les compétences requises.

Ces orientations sont formulés sous forme de produits formation s'avèrent indispensables pour atteindre les finalités d'un projet ou d'une nouvelle stratégie de formation, car, elles présentent les objectifs et les conditions générales de mise en œuvre de la formation professionnelle. On peut les répartir en trois catégories énoncées ci-après :

\section{- Un produit de formation professionnelle accessible soutenue par une formation continue}

Ce produit doit être adapté aux exigences des besoins locaux, régionaux et nationaux. Les préalables à l'admission à la formation doivent se contenter à ce qui est capital pour la réussite de la formation ciblée. Il doit reposer sur des critères objectifs, quantifiables et bien structurés de manière à garantir la flexibilité d'adaptation aux besoins changeants du marché de l'emploi et de permettre aux usagers, quels que soient leurs âges, leurs niveaux d'études ou leurs statuts, de faire valider les acquis de leurs expériences professionnelles pour obtenir un diplôme, un titre ou un certificat de qualification professionnelle.

Le savoir-faire et les connaissances obtenus doivent être régulièrement ajustés et mis à jour en fonction des nouvelles connaissances et de l'évolution technique. Il y a donc, nécessité de renforcer ces acquis avec la mise en place de différents modes d'apprentissage et d'organisation de façon continue, favorisant l'offre de formations variées. Les produits de formation doivent être adaptés aux usagers apprenants, jeunes et adultes, que soit dans les établissements de formation professionnelle ou dans les entreprises. Ces produits sont conçus de manière à faciliter l'évaluation et la production de moyens privilégiant la validation des acquis de l'expérience (VAE).

\section{- Un produit de F.P fonctionnel et polyvalent}

Par formation fonctionnelle, en entend toute formation permettant d'assurer aux usagers apprenants les capacités requises pour s'acquitter de leurs tâches professionnelles avec compétence et efficacité. Cette formation être corroborée doit par des produits formation accommodée à la nouvelle réalité économique, en adéquation avec la réalité et le devenir d'un métier. Ces produits d'apprentissage doivent être particulièrement liés à la maitrise d'un métier. Ils visent l'accomplissement de tâches, en faisant appel à de nouvelles façons de penser et d'agir en éducation qui peuvent bouleverser certaines croyances et pratiques en vigueur.

Le DFP devrait également élaborer des produits de formation polyvalente avec la collaboration des opérateurs économiques, amenant d'une part, à préparer les usagers à la mobilité, et, d'autre part, à l'exercice de fonctions suffisamment larges pour leur permettre de s'adapter de manière adéquate à un environnement de travail en constante évolution. La particularité de ces produits de formation est de faciliter l'apprentissage et le transfert de connaissances et d'habiletés afin de les utiliser dans des contextes divers. La polyvalence s'ajoute au caractère

12 Marc-André Nadeau 1988 "L'évaluation de programme" Théorie et pratique, 2 ème édition, p 183-184 
fonctionnel de la formation professionnelle, qui implique des dimensions concrètes, pratiques et directement liées à l'exercice d'un métier.

La polyvalence est un prolongement de la formation professionnelle fonctionnel, elle implique des dimensions nettement liées à l'exercice d'un métier et contribue à élever le niveau de qualification et de compétences des usagers.

\section{- Un produit de formation professionnelle coproduit avec différents partenaires \\ $\mathrm{La}$ pertinence de ce principe doit s'appuyer sur un partenariat étroit} entre les instances compétentes publiques et privées, acteurs de la société civile et du monde de l'entreprise, organismes et associations professionnels. La collaboration des partenaires permet de faire ressortir les besoins découlant de la situation actuelle, trouver une approche équilibrée et intégrer les partenaires dans le processus de réalisation des produits formation. Ces produits, ainsi conçus reflètent les besoins et les aspirations de leurs concepteurs, ne peuvent que contribuer au succès de ce concept.

Pédagogiquement parlant, les établissements de formation professionnelle doivent être ouverts aux changements et s'efforceront constamment d'adapter leurs stratégies de formation aux apprenants à travers des méthodes innovantes et un éventail de matériel didactique ayant un lien avec le monde concret est réel, ainsi que des moyens d'évaluation.

\subsection{Le cadre conceptuel de la formation en tant que produit}

Le produit final d'une analyse de besoins de formation est une description précise du type de formation qui est adaptée à la situation actuelle des professionnels et du milieu dans lequel ils évoluent. L'analyse de besoins de formation rend ainsi possible la transformation des besoins recensés en objectifs d'apprentissage, ceux-ci pouvant ensuite être réalisés dans le cadre d'activités de formation adéquates (McConnell 2003) ${ }^{13}$.

Les exigences des entreprises ont modifié les modes de transmission, d'acquisition et d'évaluation des compétences. Ils sont impliqués davantage dans l'élaboration des produits de formation pour définir leurs propres critères de compétences. Les opérateurs économiques estiment que la fonction formation néglige les enjeux et les préoccupations des entités opérationnelles. Elle donne l'impression qu'elle se laisse enfermée dans sa tour d'ivoire, avec des offres qui n'ont aucun rapport avec la réalité du marché du travail. Ainsi, le concept de l'utilité de la formation parait flou, ils attendent de la formation qu'elle puisse les aider à faire une différence significative, pour résoudre les problèmes auxquels ils sont confrontés et réussir leurs objectifs.

L'efficacité et la rentabilité économique, deux termes qui commencent à s'émouvoir dans le secteur de la formation rattrapés par le modèle entrepreneurial, ce qui incite les acteurs de la formation à maitriser le processus de production de leurs offres.

\subsection{Conception du produit formation}

Le savoir doit être appréhendé en lien avec ses usages, ainsi, la conception d'un projet concret de formation ne se résume pas à la définition d'un contenu pédagogique ou de stage, mais exige de tenir compte simultanément de la demande de l'organisation et son environnement. Il s'agit d'élaborer une démarche concrète qui répond aux différentes dimensions d'une situation de manière à s'assurer qu'elle remplissait bien le mandat crucial qui lui était conféré à cet égard. Au Maroc, les opérateurs économiques sont unanimes sur le besoin d'une formation mutante qui s'adapte davantage avec le marché du travail.

Ayants passé beaucoup de temps, à interpréter et digérer les réformes répétitives du système de la formation professionnelle marocain pour en saisir le fonctionnement, nous estimons que l'emphase était placée sur la description des objectifs opérationnels des enseignements en passant outre les finalités de la formation qui sont les énonciations des options politiques en réponse de l'analyse de besoins ressentis ou prévisibles. Les raisons de ces faiblesses sont liées à l'incertitude des débouchés et au flou des finalités qui sont éloignées de toute professionnalisation et basées sur des stratégies dépassées que nous pouvons résumer comme suit :

la conception du produit ne prend pas en compte les contraintes de qualité et les besoins du milieu de travail, (tous les opérateurs sont conscient de cette problématique mais la réalité sur le terrain est toute autre), d'une part, et, d'autre part, les hypothèses de succès ne sont pas fiables ce qui engendre un échec d'ordre plutôt technique dû au manque d'une définition claire et commune des finalités de la formation, cet aboutissement incite les autorités de tutelle à réformer le système.

Le produit répond aux besoins du moment, c'est-à-dire aux besoins à court terme, mais sa fiabilité n'est pas satisfaisante. Dans ce cas, la pertinence du produit est jugée inferieure aux attentes parce que son cycle de vie était négligé, il y a des programmes encore en usage sont périmés par rapport aux évolutions récentes du

${ }^{13}$ McCONNELL, John H. (2003), How To Identify Your Organization's Training Needs, p 223 
marché du travail, ces programmes très spécialisés préparent les usagers à des emplois dans des domaines saturés, en mettant l'accent sur l'acquisition des connaissances plutôt que de compétences et de savoir-faire. Suite aux résultats mitigés de cette stratégie, les responsables décident encore une fois de réformer le système, ce qui explique d'ailleurs les multiples réformes entreprises ces dernières années.

Un autre problème réside cette fois dans le manque de compétitivité des établissements de FP et particulièrement les EFPP, qui trouvent des difficultés à commercialiser leurs produits de formation à cause d'un mode de distribution inadapté, mauvaise réputation, incompétence des gérants, conflit d'image de marque, cannibalisation d'autres produits, etc., ce qui provoque la fermeture de quelques établissements (DFP 2014) ${ }^{14}$.

A notre avis, cette situation est principalement due à un manque de clarté des rôles et des responsabilités des cadres chargés de la gouvernance du système, manque de cohérence et de pertinence par rapport aux finalités, inadéquate des besoins et une mauvaise définition des exigences, ainsi que la faiblesse des compétences de base des usagers.

Nous estimons que la réussite d'une formation, se joue à la fois avant, pendant et après la formation.

Avant la formation : Les concepteurs des produits formation doivent procéder à un travail en amont de la formation pour développer les compétences de base des apprenants et les aider à s'impliquer davantage dans l'action de la formation et comprendre les mots clefs et très techniques en leur proposant un travail préliminaire et des premières pistes de réflexion.

Pendant la formation : L'organisme d'évaluation et les gérants d'EFP doivent privilégier le soutien d'approches qui permettent la mise en pratique dans des situations réelles en vue de gérer le rendement est de donner une rétroaction continue pour être motivée

Après la formation : Le suivi post-formation doit devenir une priorité pour l'employeur. Il permet une transposition optimale dans la vie quotidienne et sur le poste de travail pour ancrer la connaissance et la transformer en compétence. Sans ancrage mémoriel et cognitif, la courbe de l'oubli est rapide, plus de $90 \%$ des connaissances non utilisées seront oubliées dans le mois qui suit.

La conception d'un produit formation doit prendre en considération des enjeux relatifs à l'innovation, la professionnalisation, les compétences, l'adéquation entre la formation et l'emploi, la marchandisation, l'ingénierie, la valeur ajoutée, la qualité, etc. Ces mots reviennent souvent lorsqu'on évoque la formation, il s'agit en l'occurrence d'une condition sine qua non pour répondre aux besoins du marché du travail et contribuer à sa croissance et à sa productivité d'une part, et d'autre part, assurer la compétitivité des établissements de formation dans un marché de plus en plus concurrentiel.

Le maitre-mot demeure la professionnalisation et le développement des compétences. Dans cet environnement en mutation, la professionnalisation ne cesse d'attiser des débats au cours des dernières années entre toutes les parties concernées à un moment où, l'enseignement en général se rapproche du monde professionnel pour assurer un meilleur positionnement compétitif.

\section{Professionnalisation de la formation}

Le terme «professionnel» a pris une envergure qui dépasse le secteur industriel, « mais on peut d'ores et déjà indiquer que parmi les organisations concernées se trouvent celles du secteur hospitalier, de l'enseignement et la majorité des grandes firmes (ou cabinets) de conseil aux entreprises ».(Jean GADREY, 1994) ${ }^{15}$.

Ainsi les enjeux cités plus haut, nous incitent à méditer dans un premier temps sur le concept de professionnalisation dont la tendance s'est accrue et demeure une priorité majeure en terme de gestion de compétences et d'anticipation des besoins pour les entreprises, les personnes et les établissements de formation.

Pour les entreprises, se professionnaliser veut dire s'adapter à un environnement de plus en plus concurrentiel aux exigences accrues de leur secteur d'activité et aux attentes spécifiques de leurs partenaires. Pour les personnes (demandeurs d'emploi, jeunes ou seniors, salariés du secteur public ou du privé), être «professionnel ${ }^{16}$, c'est posséder les savoirs fondamentaux, les techniques de raisonnement et d'analyse, qui légitimeront l'évolution des pratiques dans des conjonctures instables, de choisir les informations appropriées et de consolider de nouvelles connaissances. Enfin, pour les établissements de formation qui évoluent dans le spectre d'une concurrence ardue, la question de la professionnalisation des formations renvoie à la relation des établissements de formation avec l'environnement économique et le milieu professionnel ainsi que l'amélioration de la qualité des dispositifs de formation. Les finalités de la formation n'ont pas cessées d'évoluer. Le terme formation est devenu aujourd'hui plus courant que l'éducation obéissant à une perception utilitaire qui se décline vers le professionnalisme. La formation fait souvent allusion aux termes,

\footnotetext{
${ }^{14}$ Direction de la formation professionnelle 2014, Rapport d'activité annuel

${ }^{15}$ Jean GADREY, (1994) «Rationalisation industrielle ou rationalisation professionnelle? p. 163

${ }^{16}$ Le terme " professionnel » utilisé ici s'applique à tous les individus exerçant une profession ou un métier et dont les besoins de formation peuvent être étudiés
}

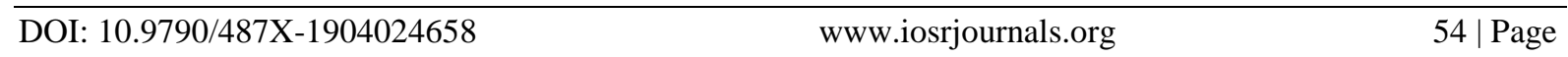


«Professionnalisation », «parcours professionnalisant», «professionnaliser », qui sont devenus, une sorte de gage de légitimité des actions de formation ce qui implique d'articuler le monde de l'éducation à celui de l'économie.

Selon G. Le Boterf (1996) «Professionnaliser» indique « le processus permettant de construire et développer ses compétences ». Pour R. Wittorski $(2007)^{17}$ professionnaliser c'est « engager un ancrage plus fort des actions de formation par rapport aux situations de travail». Il estime que «les enjeux portés par les organisations concernent l'intention d'accompagner la flexibilité du travail, la modification continue des compétences en lien avec l'évolution des situations de travail» ${ }^{18}$. La professionnalisation selon Wittorski est organisée sur trois étapes :

\section{Les trois temps de la professionnalisation}

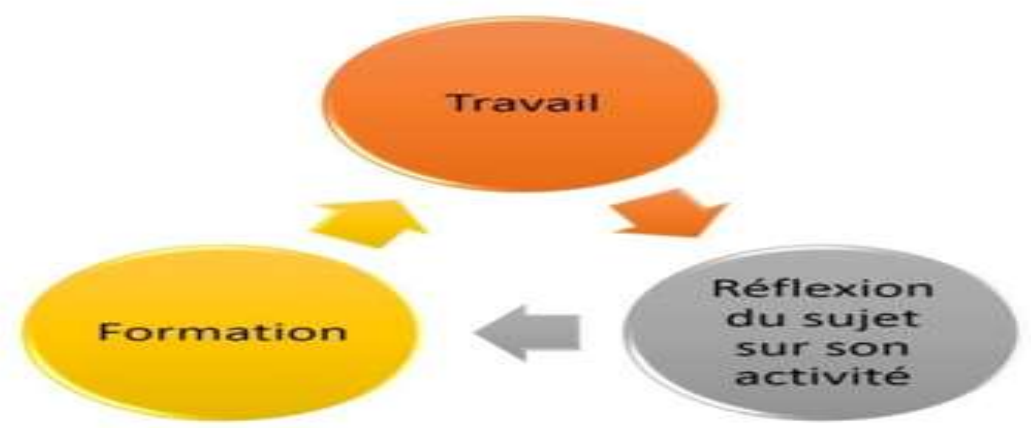

Source : Wittorski, la professionnalisation

Ce schéma de R. Wittorski, montre que la professionnalisation se rapporte aux logiques compétences du côté de l'organisation. Alors que, du point de vue individuel se décline vers la logique de la qualification. La professionnalisation conquit donc en même temps l'accroissement des compétences et une reconnaissance professionnelle. Parallèlement, la formation est devenue un métier qui s'organise, s'affine, s'industrialise, brièvement se professionnalise et fait valoir son utilité par la spécificité de ses savoir- faire, ce qui implique de nouvelles exigences de compétitivité et nécessite une connaissance approfondie et raisonnée des enjeux économiques.

Au Maroc, le champ de la formation et de l'emploi évolue rapidement. Divers facteurs sont à la base de ces évolutions. Il s'agit notamment de la décentralisation ou la régionalisation promulgué par la nouvelle constitution du 29 Juillet 2011, et de la réforme de la formation professionnelle.

Ainsi, la notion de " professionnalisation " recèle diverses finalités :

$\checkmark \quad$ La formation joue un rôle important dans l'édification des compétences qui permettent aux personnes d'agir comme de véritables " professionnels ".

$\checkmark \quad$ une édification sociale qui admet l'identification des emplois et du champ professionnel, l'accompagnement des qualifications et des compétences (référentiels des métiers, accès à l'emploi, contenu et validation d'action de formation).

$\checkmark \quad$ la motivation et la capacité des EFPP à s'organiser.

La nouvelle stratégie de la formation professionnelle 2021, lancée en 2016 vise trois finalités principales faisant référence à la professionnalisation :

$\checkmark$ développer les compétences des usagers

$\checkmark$ Préserver l'expérience professionnelle des individus et accroître leurs qualifications pour faire durer leur mission ou affermir leur " employabilité "

$\checkmark$ Préparer la conversion de leurs pratiques professionnelles.

\subsection{La formation, moteur de l'efficacité professionnelle}

La formation contribue à la professionnalisation des personnes et des organisations. Toutefois, pour participer efficacement à leurs professionnalisation, les acteurs de la formation professionnelle doivent eux aussi se professionnaliser dans le but d'acquérir une certaine maturité dans l'élaboration des produits formations susceptibles de répondre aux attentes des usagers et du milieu de travail d'une part, et, d'autre part, améliorer

\footnotetext{
${ }^{17}$ Richard Wittorski. (1997) Évolution de la formation et transformation des compétences des formateurs. pp.59 `a 72.

${ }^{18}$ Richard Wittorski. la professionnalisation. Revue Savoirs, 2008, 17, pp.11 -38.

DOI: 10.9790/487X-1904024658 www.iosrjournals.org 
leur compétitivité dans un marché où les attentes des consommateurs sont de plus en plus pressantes, ce qui leur permet de proposer des produits formation professionnalisante qui auront des retombées importantes sur leurs images de marque et deviennent un atout de prospection non négligeable tout en assurant une meilleure reconnaissance aussi bien des apprenants que des entreprises.

Les enjeux liés à la formation professionnelle ont bouleversé les méthodes traditionnelles de formation. La conception d'un bon produit formation est tributaire donc de deux facteurs :

- la professionnalisation des gérants d'établissements de formation et des formateurs

- La compétence de ces deux acteurs à maitriser les trois niveaux de compétences (savoirs, savoir-faire, savoir-être) et les trois niveaux d'action du formateur (macro, méso, micro).

\subsection{Pilotage du produit formation}

Le système de formation professionnelle doit être fondé sur une double gestion : une gestion structurée et une gestion pédagogique.

La gestion structurée: est composée de l'ensemble des éléments qui servent de définir une politique nationale de formation professionnelle, de la faire évoluer, d'articuler, d'administrer et d'établir un cadre légal et réglementaire, d'assurer la mise en œuvre de la formation ainsi que l'évaluation de la performance de l'ensemble du système.

La gestion pédagogique : quand a elle est axée sur les dispositifs et les méthodes menant à la conception, à l'élaboration et à la réactualisation continue des programmes de formation.

Pour se faire, la création d'un organisme chargé de la formation professionnelle autonome et disposant des moyens financiers s'avère une nécessité pour jouer un rôle moteur au niveau de la gestion et d'innovation pédagogique. Il doit être en lien avec les milieux de travail et les centres de formation. Son tour de table doit réunir des enseignants ou formateurs détachés ou mis à sa disposition, des salariés sous contrat ou employés occasionnellement, présentant des profils très différents, des responsables des ressources humaines, des représentants des collectivités, etc. Cette diversité apportera évidemment à la fécondité des échanges, des recherches et des ajustements lorsque cela s'avère nécessaire. Il sera donc en mesure d'élaborer une stratégie de restructuration du secteur de la formation basée sur trois angles à savoir :

- $\quad$ Etude et analyse du marché du travail,

- Enrichissement et développement des programmes de formation selon le besoin du marché du travail

- Pilotage et mise en œuvre de la formation en concentration avec les établissements de formation professionnelle et les entreprises.

Le processus d'élaboration du produit formation professionnelle doit être articulé à partir de trois composantes principales; la planification, la conception et production et l'application et suivi :

Tableau 5. Processus d'élaboration des produits de formation professionnelle

\begin{tabular}{|c|c|}
\hline Planification & $\begin{array}{l}\text { Avantage potentiel, priorité et plan général de développement à la formation professionnelle } \\
\text { - } \quad \text { Description des secteurs de formation } \\
\text { - } \quad \text { Études préliminaires } \\
\text { - } \quad \text { Orientations du développement des produits (programmes, référentiels, curriculums) }\end{array}$ \\
\hline Conception et production & $\begin{array}{ll}\text { - } & \text { Analyse du marché de travail } \\
\text { - } & \text { Définition des finalités du projet de formation } \\
\text { - } & \text { Malidation du projet de formation } \\
\text { - } & \text { Accompagnement } \\
\text { - } & \text { analyse de la rentabilité des produits }\end{array}$ \\
\hline Application et suivi & $\begin{array}{ll}- & \text { Expérimentation, s'il y a lieu } \\
\text { - } & \text { Approbation du programme par l'autorité de tutelle } \\
\text { - } & \text { Implantation } \\
\text { contrôle et suivi préventif }\end{array}$ \\
\hline
\end{tabular}

Source : moi-même

\subsection{Enjeux et défis}

Incontestablement, la formation occupe une place privilégiée dans le cadre d'un cheminement de développement qui marque l'histoire de l'humanité. Elle s'intéresse tout autant au développement de la personne qu'aux contraintes de développement économique et social.

Ce double objectif met la formation professionnelle au cœur des débats, et l'importance consacrée à ces deux pôles incite les pays à définir les orientations à suivre et de veiller à leurs mises en ouvre, tout particulièrement lors de l'élaboration des produit formations qui doivent prendre en considération des enjeux relatifs à l'innovation, la professionnalisation, les compétences, l'adéquation entre la formation et l'emploi, la

DOI: $10.9790 / 487 X-1904024658 \quad$ www.iosrjournals.org $\quad 56 \mid$ Page


marchandisation, l'ingénierie, la valeur ajoutée, la qualité, etc. Ces mots reviennent souvent lorsqu'on évoque la formation, il s'agit en l'occurrence d'une condition sine-qua-non pour répondre aux besoins du marché du travail et contribuer à sa croissance et à sa productivité d'une part, et d'autre part, assurer la compétitivité des établissements de formation dans un marché de plus en plus concurrentiel.

La formation se trouve donc face à des défis de taille résultant d'une accélération probable de la recomposition social et économique par suite de la mondialisation, de l'ouverture des échanges, des effets de la révolution des TIC, du développement de l'économie de la connaissance des individus.

En outre, chacun de ces défis représente une opportunité de développement à long terme particulièrement pour les EFPP s'ils parviennent à innover et à adapter leurs produits, leurs services et leurs pratiques de gestion, notamment en ce concerne le marketing avec des produits de première qualité qui déboucheront sur une formation ciblée répondant aux besoins des usagers et du milieu de travail pour capitaliser sur l'important potentiel non exploité du marché de la formation et d'alimenter un bouche-à-oreille favorable.

\subsection{Gérer un EFPP comme une entreprise}

Dans le secteur privé, la formation est dispensée par des organismes ou établissements de formation, ayant comme activité principale la formation des usagers en échange d'un bien. En un mot, ils vendent des places pédagogiques à des clients. Un EFPP est effectivement une entreprise qui fonctionne comme tout autre entreprise vis à vis de ses clients, il achète, vend et ses comptes doivent être équilibrés à la fin de chaque année. Pour mieux comprendre de quoi il s'agit, on va emprunter la comparaison faite par D. van Adelsberg et A. Trolley entre la fonction formation «traditionnelle » et «l'entreprise de formation »: (David Van Adelsberg, Edward A Trolley, (1999) ${ }^{19}$

\begin{tabular}{|l|l|}
\hline Fonction formation traditionnelle & Entreprise de formation \\
\hline$-\quad$ Contenus & $-\quad$ Résultats opérationnels \\
$-\quad$ Budget de formation & $-\quad$ Retour sur investissement \\
$-\quad$ Compétences & $-\quad$ Performance \\
$-\quad \begin{array}{l}\text { Nombre d'heures, coûts } \\
\text { Participants }\end{array}$ & $-\quad$ Marchés \\
\hline
\end{tabular}

En effet, les clients de la formation sont composés des :

- usagers qui souhaitent décrocher un diplôme ou développer les compétences dont ils ont besoin pour participer à l'économie en tant qu'acteur actif.

- Les entreprises qui attendent que la formation les accompagne pour améliorer leurs performances et être capables de mieux faire leur métier d'aujourd'hui et se préparer aux évolutions de l'emploi.

En effet, si le client premier d'un établissement de formation est l'usager apprenant à la recherche d'une formation initiale, les entreprises, les salariés en activité, les déflatés des services publics, etc. sont des clients secondaires des EFPP. Les EFPP doivent donc définir une politique d'ensemble de leurs interlocuteurs.

La prise en compte de cette distinction n'est pas neutre dans la mesure où les comportements d'achat de la formation, sont sensiblement différents selon ces populations. Une entreprise par exemple, va être attentive à des critères propres de son activité et à ses besoins en compétences. Le dirigeant de l'EFPP doit ici répondre aux besoins de l'entreprise avec une formation pertinente générant une plus-value pour l'entreprise. Mais aussi, il cherchera à bâtir des relations à long terme entre son établissement et l'entreprise. Ces relations sont bien entendu basées sur un avantage mutuel. L'usager particulier quant à lui, sera attentif aux services offerts par l'établissement, leurs qualités et la manière dont ils sont délivrés. Ils sont strictement liés au jugement qu'il se fait des prestations offertes par l'établissement, en terme de qualité et d'efficience, sans forcément l'exprimer explicitement. Ce qui ne l'empêche pas d'analyser d'autres propositions, Il fera confiance à son entourage, aux anciens lauréats, à la notoriété ou la bonne presse de l'établissement. Ces facteurs jouent un rôle important dans sa prise de décision. Il conviendra dès lors aux EFPP de définir ces rôles et leurs poids respectifs pour gagner la préférence de l'usager.

Les clients représentent un marché pour la formation où elle doit s'en étendre et s'en ouvrir pour améliorer sa qualité mais aussi et surtout parce qu'elle représente une partie cruciale du marché mondial des services.

${ }^{19}$ David Van Adelsberg, Edward A Trolley, 1999 « Exécution de la formation comme une entreprise ». p.19 
Il y a donc, une interdépendance entre EFPP et ses clients. Chacun d'eux vis au dépend de l'autre, si l'un de ces deux éléments manque, l'économie du marché ne peut fonctionner. En effet, le marché de la formation est trop vaste, le nombre des personnes intéressés par une formation initiale ou continue ne cesse de croitre et ne reflète pas toujours les besoins réels du marché

\section{Conclusion}

La concurrence qui règne à l'échelle mondiale sur le marché du travail, marquée par l'évolution rapide de technologie, les exigences sociétales, le ralentissement économique, la création de normes mondiales de régulation, la mobilité permanente des personnes et l'apparition constante de nouveaux emplois et de nouvelles palettes de compétences, ont provoqué une réelle innovation dans le domaine de la formation professionnelle avec l'introduction de nouvelles formes d'offre et de certification. Les exigences des entreprises ont désormais modifié les modes de transmission, d'acquisition et d'évaluation des compétences. Ils sont impliqués davantage dans l'élaboration des référentiels de formation pour définir leurs propres critères de compétences.

La formation est devenue l'un des thèmes essentiel des débats de la pensée économique, Cependant, toute tentative de conception des programmes formation sera voué à l'échec et ne parviendra pas à se développer de façon optimale sans une définition claire des finalités de la formation qui renvoie à la question d'analyse des besoins.

Dans cette perspective, les acteurs de la formation professionnelle sont tenus à définir les orientations à suivre et veiller à leurs mises en œuvre, tout particulièrement lors de l'élaboration des produit formations qui doivent prendre en considération des enjeux relatifs à l'innovation, la professionnalisation, les compétences, l'adéquation entre la formation et l'emploi, la marchandisation, l'ingénierie, la valeur ajoutée, la qualité, etc. Ces mots reviennent souvent lorsqu'on évoque la formation, il s'agit en l'occurrence d'une condition sine qua non pour répondre aux besoins du marché du travail et contribuer à sa croissance et à sa productivité d'une part, et d'autre part, assurer la compétitivité des établissements de formation dans un marché de plus en plus concurrentiel.

\section{Bibliographie}

[1]. Ardouin T., (2008).- « Formation tout au long de la vie et professionnalisation à l'université : le cas des métiers de la formation à l'université de Rouen $»$.

[2]. Ardouin T. (2006) Ingénierie de formation pour l'entreprise. Analyse, Concevoir, Réaliser, Evaluer. Paris : Dunod. (2ème éd.)

[3]. David Van Adelsberg, Edward A Trolley, 1999 "Running Training Like a Business: Delivering Unmistakable Value » en français «Exécution de la formation commeune enterprise»

[4]. FERNANDEZ, J. (1988). « Réussir une activité de formation », Montréal, Les Éditions coopératives Albert Saint-Martin

[5]. Jean GADREY, (1994) «La modernisation des services professionnels. Rationalisation industrielle ou rationalisation professionnelle? »In: Revue française de sociologie, 1994, 35-2. Aspects de la vie professionnelle. pp. 163-195;

[6]. Kaufman, R.E. (1982). Identifying and Solving Problems : A System Approach. (3ème éd.) San Diego, California. Universuty Associates.

[7]. Marc-André Nadeau, (1988) ; «L'évaluation de programme ; théorie et pratique » Presses Université Laval, 1988 - 430 pages

[8]. McCONNELL, John H. (2003), How To Identify Your Organization's Training Needs: A Practical Guide to Needs Analysis, New York, AMACOM.

[9]. Richard Wittorski. (1997) Évolution de la formation et transformation des compétences des formateurs. Education permanente, Paris : Documentation française, 1997, 132, pp.59`a 72.

[10]. R. Wittorski (2007), Professionnalisation et développement professionnel. Paris : L'Harmattan, 2007. Sylvie Fernandes. p. 315316.

[11]. Tyler, R.W. (1950). Basic Principles of Curriculum and Instruction. Chicago. III. 